\title{
The impact of mastication on cognition: Evidence for intervention and the role of adult hippocampal neurogenesis
}

\author{
Natalie Smith ${ }^{\mathrm{a}}$, Sophie Miquel-Kergoat ${ }^{\mathrm{b}}$ and Sandrine Thuret ${ }^{\mathrm{a}, *}$ \\ ${ }^{a}$ Department of Basic and Clinical Neuroscience, Institute of Psychiatry, Psychology and Neuroscience, \\ King's College London, The James Black Centre, London, UK \\ ${ }^{\mathrm{b}}$ Wrigley (Mars Inc.), Chicago, IL, USA
}

\begin{abstract}
Adult hippocampal neurogenesis (AHN) in the dentate gyrus of the hippocampus has been shown to affect mood, cognition, learning ability and memory. A growing area of interest is whether mastication or chewing also affects AHN. This article aims to collect the evidence from animal and human studies to ascertain whether mastication is an oral environment enrichment capable of having a modulatory effect on AHN and associated behaviors.

Current data point toward a causal relationship between masticatory ability and cognitive function. Experimental studies on mice and rats have consistently shown that impairing masticatory function results in physical and behavioral changes. A recurring theme of decreased neural stem cells proliferation in the hippocampus was seen in most of the studies found. Human population study has shown that tooth loss and masticatory difficulty are positively correlated with having greater odds of cognitive impairment. However, no causal mechanism has yet been found to explain the effects of mastication on AHN.

Further studies, especially in humans, are warranted to ascertain whether mastication could be used as a potential health intervention to slowdown cognitive decline in the aging population or delay the onset of diseases such as dementia.
\end{abstract}

Keywords: Mastication, chewing, hippocampus, neurogenesis, proliferation, cognition, memory, ageing, tooth

\section{Introduction}

The adult human brain has the capability to generate neural stem cells, which go on to divide and differentiate into neurons. These newborn neurons are generated throughout adult life in a process called neurogenesis. Neurogenesis is confined to the dentate nucleus of the hippocampus and the subventricular zone of the lateral ventricles in the human brain [1-3]. Adult hippocampal neurogenesis (AHN) involves the

${ }^{*}$ Corresponding author: Dr. Sandrine Thuret, Department of Basic and Clinical Neuroscience, Institute of Psychiatry, Psychology and Neuroscience, King's College London, The James Black Centre, 125 Coldharbour Lane, SE5 9NU, London, UK. Tel.: +44 0207848 5405; Fax: +44 0207848 5407; E-mail: sandrine.1.thuret@kcl.ac.uk. addition of over 700 new neurons per day to each hippocampus in the human brain [2], and multiple rodent studies have suggested that AHN affects cognition, learning and memory abilities as well as mood $[4,5]$.

With the implications AHN may have on mental health kept in mind, protection of AHN or perhaps enhancement may prove to be a desirable health intervention. Several environmental stimuli are in fact thought to enhance AHN, such as physical exercise and diet $[6,7]$. Interestingly, not only diet content can modulate AHN, but also meal frequency, calorie intake and texture [8]. A burgeoning area of study is in masticatory function and whether this affects AHN. The loss of teeth has also been suggested to be related to the development of senile dementia and Alzheimer's disease 
[9], with both conditions featuring loss of memory function and disturbed learning, areas that $\mathrm{AHN}$ has been hypothesised to modulate [4]. Several studies have investigated whether masticatory impairment affects AHN in mice and rat models, such as through soft-diet feeding (not requiring mastication) [10] and removal of molars [11] with another study demonstrating that a soft-diet in mice after weaning resulted in impaired AHN in later life [12]. A review of the relationship between mastication and dementia surmised that a correlation does indeed exist [13]. The aim of this article is to assemble studies from both human and animal investigations on the impact of mastication on mental health to ask whether mastication in humans is an oral environmental enrichment capable of modulating neurogenesis and associated behaviors (see Fig. 1 for a proposed relationship mechanism). Should this hypothesis be correct, mastication in humans could be investigated as a novel intervention in order to modulate AHN and impact on a range of mental health conditions.

\subsection{Adult hippocampal neurogenesis}

AHN occurs in the subgranular zone (SGZ) of the dentate gyrus (DG) in the hippocampus. Neurogenesis involves the proliferation of neural progenitor cells in the SGZ, which then go on to differentiate and migrate and integrate into pre-existing circuitry [14]. Neural progenitor cells lie in close contact with blood vessels, and it is believed that biochemical stimuli delivered from the blood affect AHN [15-17].

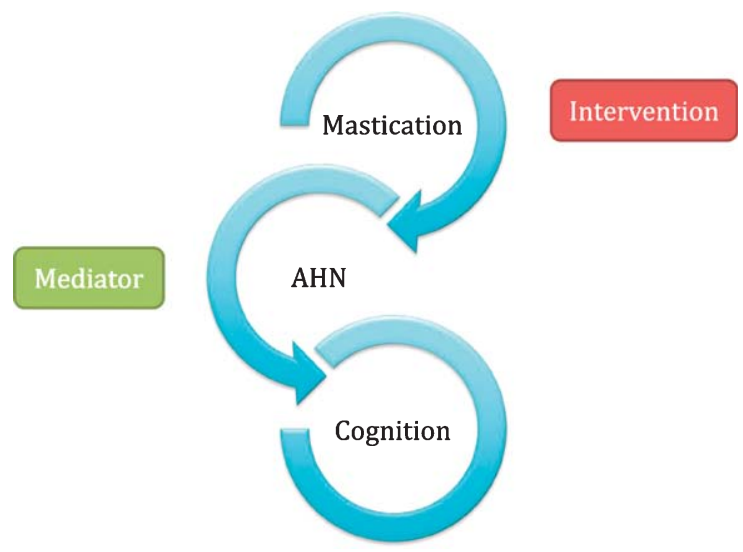

Fig. 1. The proposed hypothesis between mastication, adult hippocampal neurogenesis (AHN) and cognition. Mastication may prove to be an effective interventional strategy in humans for range of mental health conditions via modulatory effects on adult hippocampal neurogenesis (AHN).
Brain-derived neurotrophic factor (BDNF) is one of the many signaling molecules involved in regulating AHN $[14,18]$. Interestingly, it has been demonstrated in studies that soft-diet feeding in mice leads to altered expression of BDNF and its receptor, Tryosine receptor kinase $\mathrm{B}$ (TrkB), in the hippocampus and restrained AHN, which resulted in behavioral impairments [12, 19]. Impairment of this process results in impaired memory function, and studies involving ablation of these neurons demonstrates this. AHN is clearly an important neural function believed to play a role in memory and cognition, although the precise mechanisms underlying this are not yet fully explained [20].

\subsection{Modulation of AHN}

Enriched environment and undertaking exercise has been shown to promote the synthesis of BDNF and other local growth factors, which leads to enhanced neurogenesis [7, 21-23]. Calorie restriction, intermittent fasting and dietary content and their effects on neural plasticity, including AHN, in animal and human studies have been reviewed [6], with it concluded that these dietary factors have been demonstrated to promote neural plasticity. However, the mechanisms underlying these perceived effects remain to be elucidated, but in the meantime, placing importance on dietary content, frequency and amount could be a cost-effective way of preventing and ameliorating cognitive decline and mood disorders $[6$, 24]. Conversely, negative regulators of AHN include stress, chronic sleep deprivation, ageing, inflammation and depression [25-28]. Discovering the positive modulators of AHN to offset such conditions that negatively impact learning and memory could prove an important new treatment option.

\subsection{Mastication}

A loss of teeth has been recognised as a risk factor for developing dementia earlier and Alzheimer's disease [13]. The precise mechanism by which mastication is thought to exert an effect on AHN is not known. A review has discussed how exercise has been shown to increase AHN, and the act of mastication increases heart rate and cerebral blood flow, suggesting that mastication is an activity that can be considered to be physical exercise, resulting in neurogenesis [13]. It is also discussed that effects from an impaired masticatory ability on AHN may be due 
Table 1

Results from animal studies on mastication and adult hippocampal neurogenesis related measures

\begin{tabular}{|c|c|c|c|}
\hline Reference & Animal Model & Intervention & Outcome \\
\hline$[10]$ & Wistar rats & Soft diet & $\begin{array}{l}\text { Cell proliferation in the dentate gyrus is suppressed } \\
\text { by soft diet feeding }\end{array}$ \\
\hline [19] & C57BL/6 mice & Soft diet & $\begin{array}{l}\text { Soft diet mice showed behavioural impairments, } \\
\text { such as decreased home cage activity, increased } \\
\text { open field test activity, and deficits in prepulse } \\
\text { inhibition, but no impairments in memory and } \\
\text { learning. Decreased cell proliferation in the } \\
\text { hippocampus, BDNF and Akt1 (protein kinase B) } \\
\text { gene expression }\end{array}$ \\
\hline$[36]$ & C57BL/6 mice & Hard diet & $\begin{array}{l}\text { Improved spatial learning ability of hard diet mice. } \\
\text { Forced mastication increased the survival rate of } \\
\text { the newly generated neurons in the dentate gyrus } \\
\text { but did not increase progenitor cell proliferation }\end{array}$ \\
\hline [35] & $\begin{array}{l}\text { Pregnant DDY mice and their } \\
\text { offspring }\end{array}$ & $\begin{array}{l}\text { Restraint stress } \pm \text { object to } \\
\text { chew on }\end{array}$ & $\begin{array}{l}\text { Chewing in dams during prenatal stress attenuates } \\
\text { stress-induced rise in plasma corticosterone } \\
\text { levels. Adult offspring of these dams showed } \\
\text { attenuated prenatal stress-induced learning } \\
\text { deficits and decreased dentate gyrus cell } \\
\text { proliferation }\end{array}$ \\
\hline$[33]$ & C57BL/6J mice & Liquid diet & $\begin{array}{l}\text { Liquid diet group showed impaired memory and } \\
\text { learning ability. In the hippocampus, the number } \\
\text { of pyramidal neurons and expression of } \\
\text { Tropomyosin receptor kinase B was reduced, and } \\
\text { expression of BDNF was increased }\end{array}$ \\
\hline [34] & Sprague-Dawley rats & Liquid diet & $\begin{array}{l}\text { No differences in neuronal differentiation and } \\
\text { survival of newborn neurons were seen. A } \\
\text { reduction of hippocampal neuronal proliferation } \\
\text { was found in the liquid diet group }\end{array}$ \\
\hline [11] & C57BL/6 mice & Powder diet \pm molar removal & $\begin{array}{l}\text { Reduced number and survival rate of newborn cells } \\
\text { in powder diet and molarless mice }\end{array}$ \\
\hline [9] & CD1 mice & Molar removal & $\begin{array}{l}\text { Impaired distribution, structure and neurogenic } \\
\text { capacity of newborn neurons in dentate gyrus of } \\
\text { molarless mice }\end{array}$ \\
\hline
\end{tabular}

Abbreviations: BDNF - Brain-derived neurotrophic factor.

to diminished sensory input leading to reduced cell growth, disruption of cholinergic neurotransmission or disruption of the hypothalamic-pituitary-adrenal (HPA) axis [13]. It is perhaps likely that several mechanisms are involved and results from animal and human studies attempting to elucidate the relationship between mastication and AHN will be discussed below.

\subsection{Rodent studies measures}

Table 1 shows the designs and outcomes of mastication-related rodent studies. The majority of identified rodent studies investigated the effect of mastication on neurogenesis by comparing soft or hard diet fed rodents with rodents fed a normal diet. Others looked at molarless rodents compared with rodents with intact masticatory ability, or provided their intervention group with objects to chew on.

The effect of mastication was observed in most of these studies through histological examination of the rodent brains and/or behavioural analysis. Parameters used to measure the effects on the process of neurogenesis included looking at the number/density of newborn neurons, neuronal proliferation, differentiation and survival. The majority of the studies used injections of 5-bromo-2'-deoxyuridine (BrdU), a thymidine analogue, which reacts with DNA of cells in the $\mathrm{S}$ phase of mitosis, to immunohistochemically label and quantify the proliferating cells and their survival rate. Doublecortin and other neuronal nuclear antigen were used concomitantly with proliferation markers to label neuroblasts and neurons to assess neurogenic activity. 
Another approach to study the physiological effects of mastication on the hippocampus was to investigate levels of BDNF and its receptor TrkB, which play an important role in hippocampal memory and learning by promoting neuronal differentiation and survival.

In terms of looking at hippocampal-dependent behavioural outcomes many studies used the Morris water maze task assessing spatial learning and memory. Behavioural outcomes were also measured using the passive avoidance test, a fear-aggravated test used to evaluate learning and memory.

\subsection{Human studies measures}

Table 2 shows the designs and outcomes of mastication-related human studies. At this time it is not possible to measure $\mathrm{AHN}$ in living human participants and these experimental studies on the effects of mastication in humans utilises alternative methods such as measuring cerebral blood flow or using fMRI to show brain activity during chewing and comparing ability in performing memory tasks \pm chewing.

Cross sectional population studies [29], have looked at the masticatory ability and oral health of an elderly population and assessed their mental state using the mini-mental state examination (MMSE) to identify those with cognitive impairment, and found that a correlation exists between the two. This pattern of investigation was common across many reports. Other studies have looked at the effects of chewing gum on memory or attention, measuring results using fMRI to look at brain regions activated, or word recall ability [30-32].

\section{Discussion}

\subsection{Outcome of rodent studies}

The results of the studies presented in this review point toward a causal relationship between masticatory ability and cognition. Experimental studies discussed, mainly done on mice, have consistently shown that impairing masticatory function results in both cellular and behavioural changes. A recurring theme identified is that of a decrease in neural stem cells proliferation in the hippocampus in many of the animal studies, when masticatory impairment was simulated by feeding mice a soft/powdered/liquid $\operatorname{diet}[10,19,33,34]$.
These experimental studies used soft diet fed mice/rats with a control group fed a normal, solid diet. The soft diet fed mice showed signs of decreased hippocampal function, which manifested as decreased memory and learning ability, such as measured by the passive avoidance test [33]. Cell proliferation assessed by immunohistochemically labelling progenitor cells was also found to be reduced in the DG in soft diet groups at a range of different ages, demonstrating that masticatory impairment causing potential problems with memory and cognition may not be a problem just for the elderly [10].

Patten, Moller et al. (2013) found that despite the decreased progenitor cell proliferation in the SGZ of the DG, there was neither a difference in the density of immature neuroblasts nor in cell survival in adult rats, suggesting that neurogenesis overall is not affected by soft diet feeding. They hypothesised that survival rates may be upregulated in order to compensate for this decreased cell proliferation [34]. The study results demonstrated a hypothetical compensatory mechanism for neuronal proliferation when decreased by soft diets and further study into this is warranted. If a compensatory mechanism is found to be in place, perhaps it can be enhanced in order to protect neural function in those with impaired masticatory function. Interestingly, Mitome, Hasegawa et al. (2005) found that proliferation in the SGZ of the DG was not affected by soft diet \pm molarless condition in mice but the number of surviving cells was reduced. It was postulated that the molarless condition may have exerted chronic stress on the mice and this could be responsible for the results. However, Patten, Moller et al. (2013) state that despite there being differences in cell proliferation rates in rats (their study) and the mice in this study, the use of a single injection of BrdU to measure cell proliferation may not have been sufficiently accurate, as they attempted to validate their results by additionally using the more widely expressed markers, Ki67 and PCNA.

Liquid diets were also found to increase BDNF levels and decrease TrkB levels [33]. The BDNF levels are thought to be higher due to reduced uptake by $\operatorname{TrkB}$, as expression of this receptor is reduced. The exact cause of the reduced TrkB expression is unknown, but various hypotheses are put forward; decreased mastication with the liquid diet leads to a drop in sensory input from the trigeminal and facial nerve to the somatic sensory cortex, or decreased mastication stimulates BDNF expression, and the continuous release of BDNF results in negative feed- 
Table 2

Results from human studies on mastication and the effects on cognitive function

\begin{tabular}{ll}
\hline Reference & Population \\
\hline$[29]$ & $>77$ years, Swedish population \\
{$[41]$} & $70-74$ year olds, Japanese population \\
& \\
& $\begin{array}{c}>50 \text { year olds, Korean population (excluding } \\
\text { patients at baseline with dementia or } \\
\text { stroke) }\end{array}$
\end{tabular}

[38] 60 and 65 year olds, Japanese population
$>61$ year olds, Italian population

$$
\begin{aligned}
& 20-59 \text { year olds and }>70 \text { year olds, } \\
& \text { American population }
\end{aligned}
$$

55-80 year olds, Swedish population

\begin{abstract}
44 cognitively normal and 44 cognitively impaired females, Japanese population
\end{abstract}

Experiment 1: Mean age 20 years and 5 months, Cardiff University

Undergraduates. Experiment 2: Mean age 22 years and 1 month, Cardiff University Undergraduates
Oral examination, (noting number and location of teeth), medical questionnaire and assessment of cognitive function (MMSE)

Oral examination, medical questionnaire and assessment of cognitive function (MMSE)

Oral exam, assessment of basic activities of daily living and cognitive assessment (MMSE)

Data from National Health and Nutrition Examination Survey III. Oral examination and cognitive assessments

Oral examination and cognitive function tests of episodic, semantic and working memory, visuo-spatial ability and processing speed

Cognitive impairment evaluated with Hasegawa Dementia Rating Scale (Japanese version of MMSE), activities of daily living rated, number of teeth and bite force ascertained by oral exam and masticatory ability assessed as a score of ability to chew a list of 35 foods

Experiment 1: Participants were given 15 disyllabic nouns to memorise with groups either chewing flavourless gum/not chewing gum during word learning and/or during word recall. Experiment 2: Identical method using mint flavoured gum
Outcome

People with multiple tooth loss or difficulty chewing hard food had significantly higher odds of cognitive impairment

Self-assessed impairment of masticatory ability was associated with a decline in higher brain function, including immediate verbal memory ability

Association between dental health status and cognitive function becomes more strongly correlated with increase in the number of teeth lost

Association found between cognitive function in the normal MMSE score range and the number of sound/healthy and decayed teeth

Impaired mastication affects cognitive function and contributes to senile dementia and Alzheimer's disease. Loss of teeth in particular induces pathologic changes in the hippocampus

Poor oral health is associated with worse cognitive function throughout adult life

Number of natural teeth was positively related with all cognitive tests, especially with episodic recall and recognition and semantic memory but did not influence working memory, visuo-spatial ability or processing speed

A close association found between masticatory function and cognitive status. Mastication scores of cognitively normal subjects were significantly higher than those of cognitively impaired subjects

A non-additive, facilitative effect of receiving either type of gum at any point in the experiment was found on recall levels. However chewing gum did not facilitate word recall 
Table 2

(Continued)

\begin{tabular}{llll}
\hline Reference & Population & Study & Outcome \\
\hline$[31]$ & $20-34$ year olds & $\begin{array}{l}\text { Effect of chewing on attentional } \\
\text { network test (ANT) measured by } \\
\text { fMRI }\end{array}$ & $\begin{array}{l}\text { Chewing accelerates the processing } \\
\text { speed of ANT and brain regions } \\
\text { related to motor and attention were } \\
\text { more motivated during chewing, } \\
\text { suggesting chewing can lead to } \\
\text { improvements in cognitive } \\
\text { performance }\end{array}$ \\
[30] & & $\begin{array}{l}\text { Pffects of chewing gum on neuronal } \\
\text { activity in the brain during a } \\
\text { wealthy 20-39 year olds, }\end{array}$ & $\begin{array}{l}\text { including the hippocampus, after } \\
\text { chewing trial suggesting chewing } \\
\text { accelerates or recovers process of } \\
\text { working memory }\end{array}$ \\
\hline
\end{tabular}

Abbreviations: MMSE - mini-mental state examination. fMRI - functional magnetic resonance imaging.

back on TrkB expression level [33]. The disruption in $\mathrm{BDNF} / \mathrm{TrkB}$ signalling leads to reduced induction of the MAPK cascade, which is necessary for memory and learning, and the findings suggest that the reduction of BDNF/TrkB signalling decreases the number of neuronal cells in the hippocampus, resulting in impaired memory and learning ability [33]. However, a study that also found reduced BDNF levels in soft diet fed mice did not find the mice to have defective cognitive function, but instead have affected physical and mental development, which may cause an increased vulnerability to mental disorders [19]. Yet it is noted that their results are inconsistent with other studies, which demonstrate reduced performance in cognitive tasks, with long term soft diet feeding, and propose that this is due to the study having a relatively short period of soft diet feeding in comparison [19].

A study investigating the effects of molar removal on mice AHN showed impaired distribution, structure and neurogenic capacity of neurons in the DG when measured by doublecortin and neuronal nuclear antigen, with these impairments possibly having a negative effect on learning and memory [9]. Surgical molar removal however, may have imposed chronic stress on the mice, such as postulated by Mitome, Hasegawa et al. (2005), who also investigated molar removal, with the effects of stress on the HPA axis perhaps causing the reduced AHN. The effects of stress on the HPA axis are discussed in another study, which states that exposure to stress during pregnancy alters the HPA axis of the offspring, causing learning deficits and inhibiting neurogenesis [35]. It was found that dams, chewing on a wooden dowel during induced stress by restraint resulted in a decreased stress-induced release in plasma corticosterone, with learning and memory deficits and reduced cell pro- liferation ameliorated in the DG of offspring [35]. The effect of liquid diet alone, however, has not been shown to alter the HPA axis nor increase corticosterone levels, and is not thought to contribute to decreased cell proliferation [34], yet it is interesting that the effects of induced stress by other means on AHN can be ameliorated by the act of chewing, such as suggested by the studies previously discussed. Another study looking at the positive effects of mastication, rather than the negative effects of its absence, found that neural stem cell survival in the hippocampus is increased and spatial memory is improved by forced mastication through feeding mice a hard diet [36]. A change in cell proliferation was not found as compared with the control group on a normal diet, but hippocampal volume was increased in the hard diet group. It was put forward that the mechanisms behind these results could be due to sensori-motor information from the oral cavity reaching the hippocampus and affecting the rate of neurogenesis, or the muscular contractions of mastication increasing cerebral blood flow, allowing increased entry of growth factors into the DG [36]. The study concludes with the future hope that mastication could improve or accelerate the recovery of spatial memory functions in the hippocampus, preventing senile dementia [36].

\subsection{Outcome of human studies}

Human studies demonstrated similar results, with masticatory ability being associated with cognitive impairment. In a Swedish population study of humans aged 77 and over, tooth loss and masticatory difficulty were positively correlated with having greater odds of cognitive impairment [29]. A causal association 
has not yet been found; perhaps those with cognitive impairment are unable to maintain adequate oral hygiene, or cognitive impairment increases the likelihood of having a difficulty with chewing. However, this study reports on other longitudinal studies, which found that there was no significant tooth loss associated with adults with dementia and conclude that it is not likely that impaired cognition leads to tooth loss and masticatory impairment, with results leaning in favour of a causal association in the other direction [29]. It is of note that the cohort used in this study came from a time where there were no dental services and preventative programs, and teeth were more commonly extracted, suggesting that these results may not be representative of aging populations of the future [29].

Other population studies in Japan, Korea, Italy and America have also demonstrated similar results; increased number of missing or decayed teeth is associated with higher odds of cognitive impairment [37-39]. Park, Suk et al. (2013) suggest the possibility of using the number of lost teeth as a predictive factor for cognitive impairment in adults over the age of 50 in the community with no history of dementia or stroke, with Takata, Ansai et al. (2009) suggesting the same; that dental status might be a marker of 'cognitive reserve' in younger subjects. Stewart, Sabbah et al. (2008) took a large community sample of crosssectional data in their study and warn that there may be a possibility of reverse causality in their results, such as with education as a confounding factor.

When hippocampal-dependent cognitive functions and tooth loss was investigated, it was found to uniquely contribute to a significant variation in episodic and semantic memory performance, but an association between working memory, visuo-spatial ability and processing speed was not found [40]. This is yet another study with results adding to the accumulating evidence that there is a relationship between hippocampal-dependent cognitive functioning and number of teeth, however they state that longitudinal analyses are needed to shed further light on the potential causal relationship [40]. Miura, Yamasaki et al. (2003) also state that longitudinal studies are indicated in order to provide additional pertinent information, as the majority of studies done are epidemiological, cross-sectional studies. It is clear there is substantial evidence pointing towards a causal relationship from cross-sectional studies and further investigations need to establish precisely how this works and how it can be used with respect to health.
Several experimental studies have looked at the effect of chewing gum on cognitive function, to ascertain whether the act of mastication has a positive or enhancing effect on functions such as memory. The effects of chewing gum on memory and attention performance tasks have demonstrated differing results across studies. Johnson and Miles (2008) have found that chewing gum \pm mint flavour failed to produce a context-dependent memory effect, though chewing gum at some point during their word learning and recall experiment has a facilitative effect on recall levels. Hirano, Obata et al. (2008) demonstrated results that show chewing accelerates or recovers working memory and induces an arousal effect, which consequently enhances cognitive performance. A later study showed that brain regions related to motor and attention were more activated during chewing, and this increased arousal levels and alertness, which therefore could lead to improved cognitive performance [31]. These potential effects on cognitive performance by mastication could again be explained by the regional increases in cerebral blood flow, as postulated by some of the experimental studies in mice. Taking rodent data into account, the enhanced cognitive performance could be explained through increased AHN due to the increased blood flow and growth factor availability. Nevertheless, before to embark onto molecular and cellular analyses relevant to $\mathrm{AHN}$, the inconsistencies in results from experimental studies on chewing gum and its effect on cognition need to be addressed. Indeed it would be helpful to demonstrate whether mastication in healthy and perhaps younger age groups can have a protective effect on mental health or even an enhancing effect on functions such as memory.

\section{Conclusion}

The majority of results from the mastication studies points toward a causal relationship between mastication and cognitive function. When mastication was impaired in mice, results tended to show a reduced amount of neural stem cells proliferation in the hippocampus, demonstrated quantitatively with less labeled newborn cells present than in control groups [10, 19, 33, 34]. Studies additionally investigating behavioral changes in mice with impaired masticatory function had conflicting results, with some finding the mice to have memory and learning deficits [33], and another finding mental development to be affected but not cognitive function [19]. This 
study however only investigated the effects of soft diet feeding in the short term, compared with long-term studies, which did find cognitive dysfunction. It was also found that despite decreased neural progenitor cell proliferation in the DG, neuronal differentiation and survival was not affected, demonstrating a potential compensatory mechanism in place prolonging the survival of these neurons to make up for the decreased proliferation, or an alteration in cell cycle kinetics [34]. If such a compensatory mechanism is found, it could perhaps be manipulated and enhanced in people with masticatory dysfunction in order to protect them from reduced AHN leading to cognitive decline. Molar removal had a similar effect to soft-diet feeding in mice, with decreased distribution, structure and neurogenic capacity of neural progenitor cells in the DG [9].

Altered BDNF/TrkB expression has been found in soft diet fed mice and this is hypothesised to be due to decreased sensori-motor input due to lack of chewing, or decreased mastication stimulating BDNF secretion, which induces negative feedback to decrease TrkB expression [33]. This alteration in BDNF/TrkB expression is thought to impair memory acquisition and learning by decreasing the number of neurons in the hippocampus [33].

Inducing mastication in two of the studies found had a positive effect on hippocampal neurons in mice. Inducing stress in pregnant mice given something to chew on ameliorated learning and memory deficits in their offspring caused by a disrupted HPA axis [35]. Mice fed an autoclaved hard diet had increased neural stem cell survival, increased hippocampal volume and improved spatial memory [36]. The act of mastication is thought to exert effects on the hippocampus through increasing cerebral blood flow and allowing entry of growth factors in to the DG, or through sensori-motor input affecting the rate of neurogenesis [36]. In humans, it was found that masticatory ability/tooth loss was positively correlated with having greater odds of cognitive impairment, reflecting results depicting the possible causal relationship seen in the animal models [29, 37-39]. Investigation into this, such as with longitudinal studies would be helpful in establishing the extent to which mastication impacts on mental health, and whether this is through a mechanism involving AHN. Additionally, repeating experimental studies looking at how chewing gum impacts upon cognition would be indicated, in order to address the discrepancies found in results.

Further studies into the effect of mastication on AHN are warranted, as a pattern of causal associ- ation between masticatory impairment and AHN is emerging. Discrepancies between results need to be explained and the effects in humans need investigating. Should mastication prove to have an effective protective mechanism on AHN, ensuring those with tooth loss or decreased masticatory ability may be vital in protecting their cognitive health. With an increasing elderly population, prevention of cognitive decline and dementia will alleviate strain on health services and families. Additionally, ensuring young people have adequate masticatory function may prove important in preventing developmental problems and learning difficulties. These are all areas that would benefit greatly should further research and experimental study prove these theories correct. Furthermore, if masticatory effects on AHN could be enhanced, the potential for otherwise healthy people to boost memory, learning and cognitive function presents an exciting prospect.

\section{Conflict of interest}

N.S. and S.T. have no conflict of interest. S. M-K. is currently an employee of Wrigley (Mars Inc.) and confirms that there are no other potential conflicts of interest associated with this publication and there has been no significant financial support for this work that could have influenced its outcome.

\section{References}

[1] Eriksson PS, Perfilieva E, Bjork-Eriksson T, Alborn AM, Nordborg C, Peterson DA, et al. Neurogenesis in the adult human hippocampus. Nat Med 1998;4(11):1313-17.

[2] Spalding KL, Bergmann O, Alkass K, Bernard S, Salehpour M, Huttner HB, et al. Dynamics of hippocampal neurogenesis in adult humans. Cell. 2013;153(6):1219-27.

[3] Ernst A, Alkass K, Bernard S, Salehpour M, Perl S, Tisdale J, et al. Neurogenesis in the striatum of the adult human brain. Cell. 2014;156(5):1072-83.

[4] Deng W, Aimone JB, Gage FH. New neurons and new memories: How does adult hippocampal neurogenesis affect learning and memory? Nat Rev Neurosci. 2010;11(5):33950.

[5] Eisch AJ, Petrik D. Depression and hippocampal neurogenesis: A road to remission? Science. 2012;338(6103):72-5.

[6] Murphy T, Dias GP, Thuret S. Effects of diet on brain plasticity in animal and human studies: Mind the gap. Neural Plast. 2014;2014:563160.

[7] Voss MW, Vivar C, Kramer AF, van Praag H. Bridging animal and human models of exercise-induced brain plasticity. Trends Cogn Sci. 2013;17(10):525-44. 
[8] Zainuddin MS, Thuret S. Nutrition, Adult hippocampal neurogenesis and mental health. Br Med Bull. 2012;103(1): 89-114.

[9] Su S, Qi T, Su B, Gu H, Wang J, Yang L. Tooth loss inhibits neurogenesis in the dentate gyrus of adult mice. Neural Regen Res. 2014;9(17):1606-9.

[10] Aoki H, Kimoto K, Hori N, Toyoda M. Cell proliferation in the dentate gyrus of rat hippocampus is inhibited by soft diet feeding. Gerontology. 2005;51(6):369-74.

[11] Mitome M, Hasegawa T, Shirakawa T. Mastication influences the survival of newly generated cells in mouse dentate gyrus. Neuroreport. 2005;16(3):249-52.

[12] Yamamoto T, Hirayama A, Hosoe N, Furube M, Hirano S. Soft-diet feeding inhibits adult neurogenesis in hippocampus of mice. Bull Tokyo Dent Coll. 2009;50(3):117-24.

[13] Weijenberg RAF, Scherder EJA, Lobbezoo F. Mastication for the mind-The relationship between mastication and cognition in ageing and dementia. Neuroscience \& Biobehavioral Reviews. 2011;35(3):483-97.

[14] Zhao C, Deng W, Gage FH. Mechanisms and functional implications of adult neurogenesis. Cell. 2008;132(4): 645-60.

[15] Palmer TD, Willhoite AR, Gage FH. Vascular niche for adult hippocampal neurogenesis. J Comp Neurol. 2000;425(4): 479-94.

[16] Murphy T, Thuret S. The systemic milieu as a mediator of dietary influence on stem cell function during ageing. Ageing Res Rev. 2015;19:53-64.

[17] Spencer JP. Beyond antioxidants: The cellular and molecular interactions of flavonoids and how these underpin their actions on the brain. Proc Nutr Soc. 2010;69(2):244-60.

[18] Mu Y, Lee SW, Gage FH. Signaling in adult neurogenesis. Curr Opin Neurobiol. 2010;20(4):416-23.

[19] Nose-Ishibashi K, Watahiki J, Yamada K, Maekawa M, Watanabe A, Yamamoto G, et al. Soft-diet feeding after weaning affects behavior in mice: Potential increase in vulnerability to mental disorders. Neuroscience. 2014;263(0): 257-68.

[20] Koehl M, Abrous DN. A new chapter in the field of memory: Adult hippocampal neurogenesis. Eur J Neurosci. 2011;33(6):1101-14.

[21] Stangl D, Thuret S. Impact of diet on adult hippocampal neurogenesis. Genes Nutr. 2009;4(4):271-82.

[22] Brown J, Cooper-Kuhn CM, Kempermann G, Van Praag H, Winkler J, Gage FH, et al. Enriched environment and physical activity stimulate hippocampal but not olfactory bulb neurogenesis. Eur J Neurosci. 2003;17(10):2042-6.

[23] Kempermann G, Fabel K, Ehninger D, Babu H, Leal-Galicia $\mathrm{P}$, Garthe A, et al. Why and how physical activity promotes experience-induced brain plasticity. Front Neurosci. 2010;4:189.

[24] Maruszak A, Pilarski A, Murphy T, Branch N, Thuret S. Hippocampal neurogenesis in Alzheimer's disease: Is there a role for dietary modulation? J Alzheimers Dis. 2014;38(1):11-38.

[25] Mueller AD, Meerlo P, McGinty D, Mistlberger RE. Sleep and Adult Neurogenesis: Implications for cognition and mood. Curr Top Behav Neurosci. 2013;25:151-181.

[26] Musaelyan K, Egeland M, Fernandes C, Pariante CM, Zunszain PA, Thuret S. Modulation of adult hippocampal neurogenesis by early-life environmental challenges triggering immune activation. Neural Plast. 2014;2014:194396.
[27] Borsini A, Zunszain PA, Thuret S, Pariante CM. The role of inflammatory cytokines as key modulators of neurogenesis. Trends Neurosci. 2015;38(3):145-157.

[28] Miller BR, Hen R. The current state of the neurogenic theory of depression and anxiety. Curr Opin Neurobiol. 2015;30: 51-8.

[29] Lexomboon D, Trulsson M, Wårdh I, Parker MG. Chewing Ability and Tooth Loss: Association with cognitive impairment in an elderly population study. J Am Geriatr Soc. 2012;60(10):1951-6.

[30] Hirano Y, Obata T, Kashikura K, Nonaka H, Tachibana A, Ikehira $\mathrm{H}$, et al. Effects of chewing in working memory processing. Neurosci Lett. 2008;436(2):189-92.

[31] Hirano Y, Obata T, Takahashi H, Tachibana A, Kuroiwa D, Takahashi T, et al. Effects of chewing on cognitive processing speed. Brain Cogn. 2013;81(3):376-81.

[32] Johnson AJ, Miles C. Chewing gum and context-dependent memory: The independent roles of chewing gum and mint flavour. Br J Psychol. 2008;99(Pt 2):293-306.

[33] Okihara H, Ito J, Kokai S, Ishida T, Hiranuma M, Kato C, et al. Liquid diet induces memory impairment accompanied by a decreased number of hippocampal neurons in mice. $\mathrm{J}$ Neurosci Res. 2014;92(8):1010-7.

[34] Patten AR, Moller DJ, Graham J, Gil-Mohapel J, Christie BR. Liquid diets reduce cell proliferation but not neurogenesis in the adult rat hippocampus. Neuroscience. 2013;254(0):17384.

[35] Onishi M, Iinuma M, Tamura Y, Kubo K. Learning deficits and suppression of the cell proliferation in the hippocampal dentate gyrus of offspring are attenuated by maternal chewing during prenatal stress. Neurosci Lett. 2014;560(0):77-80.

[36] Akazawa Y, Kitamura T, Fujihara Y, Yoshimura Y, Mitome M, Hasegawa T. Forced mastication increases survival of adult neural stem cells in the hippocampal dentate gyrus. Int J Mol Med. 2013;31(2):307-14.

[37] Park H, Suk SH, Cheong JS, Lee HS, Chang H, Do SY, et al. Tooth loss may predict poor cognitive function in community-dwelling adults without dementia or stroke: The PRESENT project. J Korean Med Sci. 2013;28(10):1518-21.

[38] Takata Y, Ansai T, Soh I, Sonoki K, Awano S, Hamasaki $\mathrm{T}$, et al. Cognitive function and number of teeth in a community-dwelling elderly population without dementia. J Oral Rehabil. 2009;36(11):808-13.

[39] Mummolo S, Ortu E, Necozione S, Monaco A, Marzo G. Relationship between mastication and cognitive function in elderly in L'Aquila. Int J Clin Exp Med. 201415;7(4):1040-6.

[40] Hansson P, Sunnegardh-Gronberg K, Bergdahl J, Bergdahl M, Nyberg L, Nilsson LG. Relationship between natural teeth and memory in a healthy elderly population. Eur J Oral Sci. 2013;121(4):333-40.

[41] Moriya S, Tei K, Murata A, Yamazaki Y, Hata H, Muramatsu $\mathrm{M}$, et al. Associations between self-assessed masticatory ability and higher brain function among the elderly. J Oral Rehabil. 2011;38(10):746-53.

[42] Stewart R, Sabbah W, Tsakos G, D’Aiuto F, Watt RG. Oral health and cognitive function in the Third National Health and Nutrition Examination Survey (NHANES III). Psychosom Med. 2008;70(8):936-41.

[43] Miura H, Yamasaki K, Kariyasu M, Miura K, Sumi Y. Relationship between cognitive function and mastication in elderly females. J Oral Rehabil. 2003;30(8):808-11. 